\title{
Shell effects in Duflo-Zuker inspired mass formulas: a status report
}

\author{
César A. Barbero* \\ Departamento de Física, Universidad Nacional de La Plata, C. C. 67, 1900 La Plata, Argentina \\ Instituto de Física La Plata, CONICET, 1900 La Plata, Argentina \\ E-mail: barberodfisica.unlp.edu.ar

\section{Jorge G. Hirsch} \\ Instituto de Ciencias Nucleares, Universidad Nacional Autónoma de México, AP 70-543, 04510 \\ México, DF, México
}

\section{Alejandro E. Mariano}

Departamento de Física, Universidad Nacional de La Plata, C. C. 67, 1900 La Plata, Argentina Instituto de Física La Plata, CONICET, 1900 La Plata, Argentina

\begin{abstract}
Attempts to simplify and improve the Duflo-Zuker (DZ) mass formula are reported. The "master term", which relates the volume and surface terms in the Liquid Drop Model with the microscopic monopole interaction, is modified to incorporate directly the observed shell effects in four different ways. They are, however, less successful than the macroscopic sector of the DZ model, and than those inspired in the F-spin symmetry.
\end{abstract}

X Latin American Symposium on Nuclear Physics and Applications (X LASNPA),

1-6 December 2013

Montevideo, Uruguay

\footnotetext{
*Speaker.
} 


\section{Introduction}

Nuclear mass is one of the most fundamental properties of nuclei. The interest in studying nuclear masses is twofold. First, to know nuclear masses of different nuclear species is a necessity for astrophysics, mainly because they need to accurately know the Q-values of different nuclear reactions in order to explain the origin of the elements [四. The Atomic Mass Evaluation 2003 (AME03) [[] reports the masses of the measured 2149 species (with $N \geq 8, Z \geq 8$ ). ${ }^{1}$ However, many nuclear reactions taking part in astrophysical processes involve nuclei quite far from the stability line, whose masses have not been measured, requiring instead the best theoretical predictions. Second, because of the absence of a full theory of the nuclear interaction, to find a theoretical mass formula able to reproduce the experimental nuclear masses could shed light on the basis of the nuclear theory. Consequently, decades of work have been invested to develop several microscopic and macroscopic mass models, which have succeeded in adjusting the known masses with average deviations smaller than half an $\mathrm{MeV}$. However, a better precision is still needed in nuclear calculations, and the predictions of the different models are in general divergent [田]. Accurate theoretical predictions of nuclear masses remain a challenge.

Nuclear mass formulas are usually built with a macroscopic sector which resembles the Liquid Drop Model (LDM), and a microscopic one which includes information about shell effects. All of them fit known nuclear masses with certain deviations, weighted through the root mean square (RMS). The Duflo-Zuker (DZ) mass model []] provides one of the simplest and more precise ways to predict nuclear masses. In fact, it gives an RMS of $373 \mathrm{keV}$ with a set of 28 parameters in the original version. Another code, with only 10 parameters (DZ10) [ [6] leads to good though less spectacular results but embodies the essence of the model. This model leads to an RMS of 554 $\mathrm{keV}$.

In [ $[\mathbf{D}]$ a proposal was made to reformulate the DZ model, including the shell closures in a more direct way. It led to the exploration of the ability of the LDM to describe spherical and deformed nuclei. Challenging some of our preconceptions, we found that when the LDM is employed, the masses of prolate deformed nuclei are far better described than those of spherical ones [8], making clear, one more time, that the inclusion of shell closures is the most important challenge in the

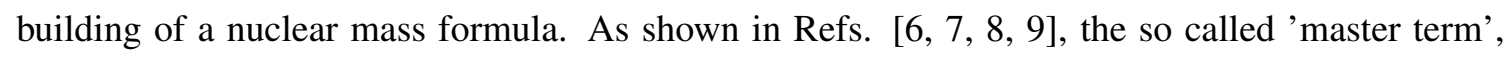
inspired in realistic interactions, reproduce the usual volume and surface terms of the LDM and is the basic building block of the DZ model. The master term in DZ model is built with harmonic oscillator (HO) shells, introducing the nuclear magic numbers through an 'ad-hoc' term. On the other side, the DZ10 model from [可] and also those developed in Refs. [ [ 8 , 团 include the magic numbers directly in the master term through the replacement of the HO closures by the ExtruderIntruder (EI) shells [प].

In this contribution we present a status report of our exploration on: i) the microscopic origin of the master term, and the way to include from the very beginning the shell closures, keeping the consistency with the realistic nuclear forces; ii) the inclusion of two, three and four body interactions through higher order terms in the number of valence nucleons. We will analyze the effect of different forms of the master and the many body interactions terms on the fits. The paper is orga-

\footnotetext{
${ }^{1}$ A new Atomic Mass Evaluation performed in the year 2012 (AME12) was very recently published [B]].
} 
nized as follows: in the second section we discuss the inclusion of shell effects through different forms of the master term, in the third section we add two, three and four body terms to the different models, and conclusions are presented in the last section.

\section{Shell effects}

We combine in a single mass formula both the ability of the LDM to describe the masses of deformed nuclei, and that of DZ to include shell effects. To this end, we will work with a LDM, adding the shell effects present in the DZ master term, but with their asymptotic behavior removed, because it is basically contained in the volume and surface terms of the LDM. The nuclear negative interaction energy is given by [8]

$$
E_{L D M+\text { shell effects }}=E_{L D M}+a_{\text {vol }}\left(M_{a}-M_{a, a s y m}\right)+a_{\text {surf }}\left(M_{a}-M_{a, a s y m}\right) / \rho,
$$

where

$$
E_{L D M}=-a_{v} A+a_{s} A^{2 / 3}+S_{v} \frac{4 T(T+1)}{A\left(1+y A^{-1 / 3}\right)}+a_{c} \frac{Z(Z-1)}{(1-\Lambda) A^{1 / 3}}-a_{p} \frac{\Delta}{A^{1 / 3}} .
$$

Here: (i) the pairing interaction is given by $\Delta=2,1$, and 0 for even-even, odd-mass and odd-odd nuclei, respectively; (ii) a correction to the radius of the nucleus is included through a modification $\Lambda$ in the Coulomb term, $\Lambda=\frac{N-Z}{6 Z\left(1+y^{-1} A^{1 / 3}\right)}$; (iii) the symmetry term employs $4 T(T+1)$, with $T=$ $|N-Z| / 2$, instead of $(N-Z)^{2}$ to account for the Wigner energy; and (iv) the Coulomb interaction is proportional to $Z(Z-1)$ to avoid the Coulomb interaction of a proton with itself.

The master term in (․] is:

$$
M_{a}=\frac{1}{\rho}\left(e_{1 v}^{2}+e_{1 \pi}^{2}\right), \quad \text { with } \quad e_{1 v}=\sum_{p_{v}} \frac{n_{v}}{G_{p_{v}}}, \quad e_{1 \pi}=\sum_{p_{\pi}} \frac{n_{\pi}}{G_{p_{\pi}}},
$$

where $n_{\pi}$ and $n_{v}$ are number operators for neutrons and protons, respectively, and $\rho=A^{1 / 3}\left[1-\left(\frac{T}{A}\right)^{2}\right]^{2}$ is the scaling factor. The denominator $G_{p}$ contains the microscopic information about the monopole sector of the nuclear interaction. Numerical studies of realistic interactions (chiral N3LO interaction) show that, when a major shell is full, the dominant isoscalar master eigenvector $U_{p}$ behaves as $U_{p} \propto D_{p}^{-1 / 2}$, being $D_{p}$ the degeneracy of the major shell of principal quantum number $p$. However, a variant $U_{p} \propto D_{p}^{1 / 2}-(2 / 15) D_{p}^{-1}$ behavior has been seen to be almost indistinguishable from the first one [6]. We have explored this freedom in the parametrization of the monopole part of the nuclear Hamiltonian in [ [6, 目, 团]. Additionally, other possible adjusts have been analyzed in Ref. [ㅁ]]. Inspired in these results, we propose here four different models:

\section{- $\underline{\text { model1 }}$}

We adopt $G_{p}=D_{p}^{1 / 2}$ from Refs. [6, [8].

- $\underline{\text { model2 }}$

We adopt $1 / G_{p}=D_{p}^{-1 / 2}-(2 / 15) D_{p}^{-1}$ from Ref. [Q] ; 
- $\underline{\text { model } 3}$

We adopt $G_{p}=\frac{D_{p}}{E_{p}^{\frac{2}{3}}-\left(E_{p}-D_{p}\right)^{\frac{2}{3}}}$ from Ref. [四], with $E_{p}=\frac{1}{3}(p+1)\left[(p+1)^{2}+5\right]$.

- $\underline{\text { model4 }}$

We adopt $G_{p}=\frac{D_{p}}{g\left(E_{p}\right)-g\left(E_{p}-D_{p}\right)}$ from [प]], with $g(X)=X^{\frac{2}{3}}\left[1-\frac{1}{2(3 X)^{\frac{2}{3}}}\right]^{-\frac{1}{2}}$.

Here $D_{p}=(p+1)(p+2)+2$ describes the EI degeneracies (which reproduce to the observed closures at $N, Z=14,28,50,82$ and 126).

Finally, the asymptotic terms in (R. $\mathbb{D}$ ) were calculated interpolating $e_{1 v}$ vs $N$ by a function of the form $c_{0}+c_{1} N^{1 / 3}+c_{2} N^{2 / 3}+c_{3} N$ [ [Q] for the set of 2149 nuclei reported in AME03. The specific expressions of these terms are given in Eq. (10) from Ref. [Q].

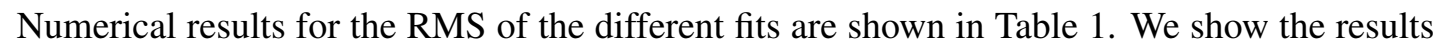
obtained when all the 2149 nuclei are included in the fit (second column) and also those obtained when only the 185 semimagic nuclei are considered (third column). From these results we can observe that all models give similar RMS values. For completeness, in Figure 1 we have compared the difference between experimental and theoretical binding energies, $B E_{\text {exp }}-B E_{t h}$, for the LDM and model2. This figure shows that shell effects are smaller but they are still there. Finally, it is important to mention that we have also explored another models using different asymptotic terms $M_{a, a s y m}$ obtained interpolating $M_{a}$ vs A, with no visible improvement in the fits.

Table 1: RMS for different models.

\begin{tabular}{c|cc}
\hline model & RMS for 2149 nuclei (in KeV) & RMS for semimagic (in KeV) \\
\hline$E_{L D M}$ & 2387 & 2113 \\
$E_{\text {model } 1}$ & 1407 & 952 \\
$E_{\text {model } 2}$ & 1402 & 942 \\
$E_{\text {model } 3}$ & 1402 & 939 \\
$E_{\text {model } 4}$ & 1403 & 940 \\
\hline
\end{tabular}

\section{Two, three and four body interactions terms}

We refine our model adding terms with higher powers on the number of valence nucleons, which describe two, three and four body interactions following the DZ ideas [प]]:

$$
\begin{aligned}
& S_{2}=\frac{n_{v} \bar{n}_{v}}{D_{p_{v}}}+\frac{z_{v} \bar{z}_{v}}{D_{p_{\pi}}}, \\
& S_{3}=\frac{n_{v} \bar{n}_{v}\left(n_{v}-\bar{n}_{v}\right)}{D_{p_{v}}}+\frac{z_{v} \bar{z}_{v}\left(z_{v}-\bar{z}_{v}\right)}{D_{p_{\pi}}}, \\
& S_{4}=\frac{n_{v} \bar{n}_{v} z_{v} \bar{z}_{v}}{D_{p_{v}} D_{p_{\pi}}}
\end{aligned}
$$



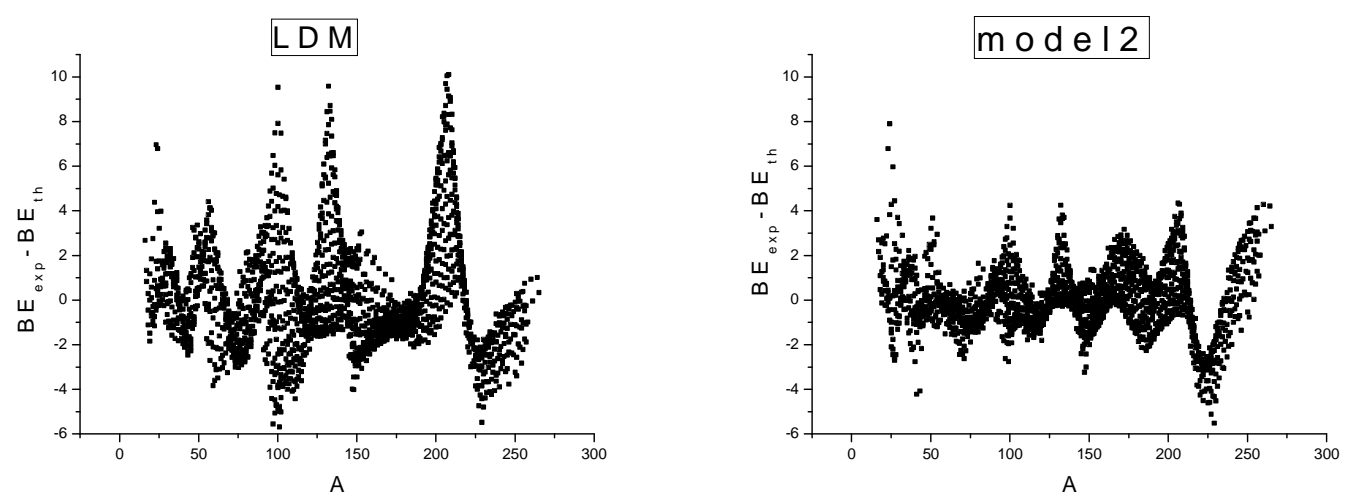

Figure 1: Comparison between LDM and model2 fits.

where $\bar{n}_{v}=D_{p_{v}}-n_{v}$ and $\bar{z}_{v}=D_{p_{\pi}}-z_{v}$ with $D_{p_{v}}\left(D_{p_{\pi}}\right)$ being the degeneracy of the neutron (proton) valence shell.

We have analyzed the effect of different combinations of these terms, including also divisions by different powers of $\rho$. Particularly, we consider interesting to analyze the following models:

- $\underline{\text { model5 }}$

We add shell corrections which are linear and quadratic in the total number of valence nucleons [ए]]

$$
E_{\text {model } 5}=E_{L D M}+b_{1}\left(n_{v}+z_{v}\right)+b_{2}\left(n_{v}+z_{v}\right)^{2} .
$$

- $\underline{\text { model6 }}$

We add many body interaction terms to our model2:

$$
E_{\text {model } 6}=E_{\text {model } 2}+a_{1} S_{2}+a_{2}\left(S_{2}\right)^{2}+a_{3} S_{3}+a_{4} S_{4} .
$$

- $\underline{\text { model7 }}$

We add many body interaction terms as in Ref. [ए]]

$$
E_{\text {model } 7}=E_{\text {model } 5}+a_{1} S_{2}+a_{2}\left(S_{2}\right)^{2}+a_{3} S_{3}+a_{4} S_{4} .
$$

We present the results for the fits using these models in Table $\square$ and Figure 2. Comparing model 2 with model5, and model 6 with model7, we conclude that:

1. Employing $b_{1}\left(n_{v}+z_{v}\right)+b_{2}\left(n_{v}+z_{v}\right)^{2}$ is more efficient that using $M_{a}-M_{a, a s y m}$ to reproduce the shell effects.

2. This last option describes better than the other the semimagic nuclei.

3. The rounded peaks at $A \sim 180$ and 270 within model 2 are due to deformation effects, which are better described (removed) within model6 and in the original DZ formalism [ [వ]. 
Table 2: RMS for different models.

\begin{tabular}{c|cc}
\hline model & RMS for 2149 nuclei (in KeV) & RMS for semimagic (in KeV) \\
\hline$E_{\text {model5 }}$ & 1075 & 1038 \\
$E_{\text {model } 6}$ & 901 & 737 \\
$E_{\text {model7 }}$ & 888 & 817 \\
\hline
\end{tabular}
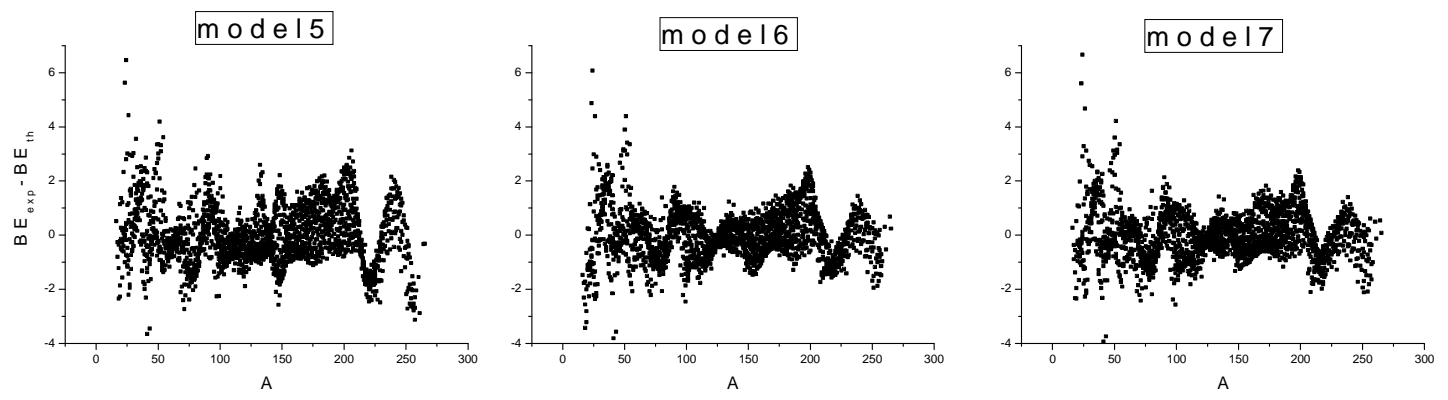

Figure 2: model5, model6 and model7 fits.

\section{Conclusions}

We have analyzed the inclusion of shell effects through different forms of the master term. Besides, we have discussed and compared different ways of adding terms accounting for two, three and four body interactions. Summarizing:

- The major challenge in going beyond the LDM description of nuclear masses is the inclusion of shell effects.

- The different ways to construct the master terms presented above allow to built a macroscopic mass formula which in some cases is able to remove the shell effects as well as DZ10, without the need of the 'ad-hoc' term.

- Employing the master terms constructed by Typel to fully enforce the linear behavior of $M_{a}$ with A allows for fits with smaller RMS than the other options explored.

- Present results seem to indicate that the strategy suggested in [ [, Q], to modify the master term using $M_{a}-M_{a, a s y m}$, is less efficient than employing the terms $b_{1}\left(n_{v}+z_{v}\right)+b_{2}\left(n_{v}+z_{v}\right)^{2}$ from Ref. [U]]. These has also been verified analyzing a model8 such us $E_{\text {model } 8}=E_{\text {model } 7}+$ $a_{v o l}\left(M_{a}-M_{a, a s y m}\right)+a_{\text {surf }}\left(M_{a}-M_{a, a s y m}\right) / \rho$ which given an $R M S=883 \mathrm{MeV}$, essentially the same as model7. 
Finally, we conclude that our detailed description of the shell effects, which includes the shell effects directly in the mast term, cannot reach the precision of the DZ10 mass formula, neither outperforms the approach based in the F-spin symmetry. The challenge to obtain a simple and strongly predictive formalism to predict nuclear masses, at the level required in nuclear astrophysics, remains open.

\section{Acknowledgments}

C.B. and A.M. are fellows of the CONICET, CCT La Plata (Argentina) and thank for partial support under Grant PIP No 0349. This work was also supported in part by Conacyt, Mexico, and by DGAPA, UNAM project IN103212.

\section{References}

[1] C.E. Rolfs and W.S. Rodney, Cauldrons in the Cosmos: Nuclear Astrophysics, University of Chicago Press (2005).

[2] G. Audi, A.H. Wapstra and C. Thibault, Nucl. Phys. A 729, 337 (2003).

[3] M. Wang, G. Audi, A.H. Wapstra, F.G. Kondev, M. MacCormick, X. Wu, and B. Pfeiffer, Chin. Phys. C 36 (2012) 16033.

[4] D. Lunney, J.M. Pearson, C. Thibault, Rev. Mod. Phys. 75 (2003) 1021.

[5] J. Duflo, Nucl. Phys. A 576 (1994) 29; A.P. Zuker, Nucl. Phys. A 576 (1994) 65; J. Duflo and A.P. Zuker, Phys. Rev. C 52 (1995) R23.

[6] J. Mendoza-Temis, J.G. Hirsch and A.P. Zuker, Nucl. Phys. A 843 (2010) 14.

[7] J.G. Hirsch and J. Mendoza Temis, J. Phys. G: Nucl. Part. Phys. 37 (2010) 064029.

[8] C. Barbero, J.G. Hirsch and A. Mariano, Nucl. Phys. A 874 (2011) 81.

[9] C. Barbero, J.G. Hirsch and A. Mariano, AIP Conf. Proc. 1488 (2012) 162.

[10] S. Typel, private communication.

[11] A.E.L. Dieperink and P. Van Isacker, Eur. Phys. Jour. A 42 (2009) 269. 